\title{
Fast Full-Wave Analysis of Multistrip Transmission Lines Based on MPIE and Complex Image Theory
}

Joaquin Bernal, Francisco Medina, Member, IEEE, Rafael R. Boix, Member, IEEE, and Manuel Horno, Member, IEEE

\begin{abstract}
The mixed-potential electric-field integral equation is used in conjunction with the Galerkin's method and complex image theory for analyzing a transmission line with multiple strips embedded in different layers of a multilayered uniaxially anisotropic dielectric substrate. The two-dimensional Green's functions for the scalar and vector potentials are analytically obtained in the space domain due to the approximation of its spectral-domain version with complex images, thus avoiding lengthy numerical evaluations. Double integrals involved in the computation of Galerkin's matrix entries are quasi-analytically carried out for the chosen basis functions, which are well suited to the problem.
\end{abstract}

Index Terms-Complex image method, integral equations, layered media, planar transmission lines.

\section{INTRODUCTION}

$\mathbf{T}$ HE analysis of a planar multistrip system such as that shown in Fig. 1 has been carried out by using a variety of techniques during the past three decades, including both quasi-TEM and full-wave formulations. Achieving high numerical efficiency has been the goal of many recent papers. A sample of this type of work in the frame of the quasi-TEM analysis can be found in [1] and references therein. In this paper, emphasis is placed on the full-wave approach. Very efficient algorithms dealing with the full-wave analysis of planar lines have been also reported, including the singular integral-equation method [2], [3] and the eigenvalue approach [4] for boxed structures, the Wiener-Hopf method [5], and various enhanced implementations of the spectral-domain analysis (SDA) [6]-[9]. In this paper, the authors propose a very fast analysis of the structure in Fig. 1 based on the mixed-potential integral equation (MPIE) [10]-[12]. The numerical performance of this approach is drastically improved by using a suitable two-dimensional (2-D) space-domain representation of the potential Green's functions and quasi-analytical computation of the reaction integrals appearing when a Galerkin scheme is used for solving the MPIE to find the surface currents. This fast and accurate computation of Galerkin's matrix entries is the key

Manuscript received July 27, 1999; revised December 10, 1999. This work was supported by the Comisión Interministerial de Ciencia y Tecnología, Spain under Project TIC95-0447.

J. Bernal is with the Department of Applied Physics, University of Seville, 41092 Seville, Spain.

F. Medina and R. R. Boix are with the Microwave Group, Department of Electronics and Electromagnetism, University of Seville, 41012 Seville, Spain (e-mail: medina@cica.es).

M. Horno, deceased, was with the Microwave Group, Department of Electronics and Electromagnetism, University of Seville, 41012 Seville, Spain.

Publisher Item Identifier S 0018-9480(00)02050-0.

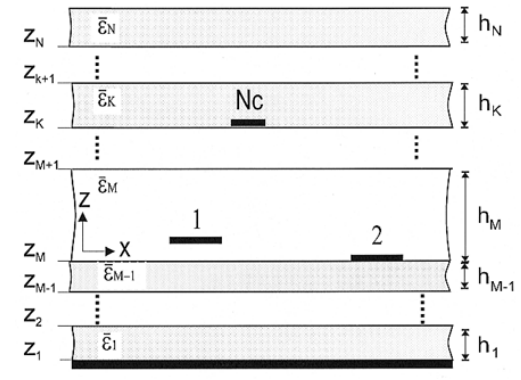

Fig. 1. Cross section of the multiconductor transmission line under analysis.

point to get very high efficiency. In this way, the determination of space-domain Green's functions is carried out via the complex images technique [13]-[15], thus avoiding commonly used numerical spectral 2-D Sommerfeld-type integration [16], [17]. This method, originally intended for the analysis of a radiating dipole in a three-dimensional (3-D) stratified medium, has been adapted here to our 2-D problem. At this point, it should be mentioned that a correction to the formulation of [15], introduced by Kipp and Chan in [18], must be also applied in the 2-D case. As it is well known, first- and second-kind Chebyshev polynomials weighed by the proper strip edge condition are very suitable basis functions for the current expansion [9]. The reaction integrals involving these functions and the closed-form expression of the Green's functions obtained with the complex images method are quasi-analytically computed. Therefore, the most time-consuming step in searching for the propagation constants, which is the computation of Galerkin's matrix, is drastically accelerated.

\section{FORMULATION OF THE INTEGRAL EQUATION}

Let us consider a transmission line consisting of $N_{c}$ infinitely thin strips embedded in the various layers of a multilayered substrate (see Fig. 1). Each layer is a uniaxial anisotropic dielectric, with its optical axis perpendicular to the interfaces between layers. Since we are interested in modes that propagate in the $y$-direction, we assume a common phase factor $e^{-j \beta y}$ for fields and currents, where $\beta$ is the unknown propagation constant. By enforcing the boundary condition for the tangential electric field at the surface of the conductors, we obtain an electric-field integral equation (EFIE). The kernel of this EFIE has a severe singularity that makes it unsuitable for a direct application of the method of moments [10]. To overcome this difficulty, we can transform this integral equation into an MPIE form, whose kernel has a weaker singularity [10], [11], [19]. 
Since the sources in our structure are perpendicular to $\hat{z}$, the MPIE has the following form:

$$
\begin{aligned}
\hat{z} \times \sum_{i=1}^{N_{c}}\left\{j \omega \boldsymbol{A}_{i}\left(x, z_{j}^{c}\right)+\right. & \left.\left(\hat{\boldsymbol{x}} \frac{\partial}{\partial x}-\hat{\boldsymbol{y}} j \beta\right) \Phi_{i}\left(x, z_{j}^{c}\right)\right\}=0 \\
& \left(x, z_{j}^{c}\right) \in L_{j}\left(j=1, \cdots, N_{c}\right)
\end{aligned}
$$

on the conducting strips. $\mathbf{A}_{i}$ and $\Phi_{i}$ in (1) are the magnetic vector potential and electric scalar potential due to the surface current on the $i$ th conductor

$$
\begin{aligned}
& A_{i}(x, z)=\int_{L_{i}} \bar{K}^{A}\left(x, z \mid x^{\prime}, z_{i}^{\prime c}\right) \boldsymbol{J}\left(x^{\prime}\right) d x^{\prime} \\
& \Phi_{i}(x, z)=\int_{L_{i}} K^{\Phi}\left(x, z \mid x^{\prime}, z_{i}^{\prime c}\right) \frac{j}{\omega}\left(\hat{x} \frac{\partial}{\partial x}-\hat{y} j \beta\right) \boldsymbol{J}\left(x^{\prime}\right) d x^{\prime} .
\end{aligned}
$$

In (1)-(3), $L_{i}$ stands for the surface of the $i$ th conductor placed at the plane $z=z_{i}^{c}\left(i=1, \cdots, N_{c}\right)$. It is well known that for a horizontally directed dipole, two components of the vector potential are necessary to satisfy the boundary conditions at the interfaces [20]. We have used the traditional Sommerfeld's formulation for the vector potential [21] so the $z$ component of the vector potential is chosen together with the component parallel to the source. This formulation is convenient for the analysis of planar structures because $K_{x y}^{A}=K_{y x}^{A}=0$. Moreover, the revolution symmetry of our problem substrate around the $z$-axis leads to $K_{x x}^{A}=K_{y y}^{A}$. Therefore, only one spectral integral is necessary for obtaining the 2-D Green's function of the magnetic vector potential.

\section{KERNEL OF THE INTEGRAL EQUATION}

It is feasible to obtain a closed-form expression for $K_{x x}^{A}\left(x, z \mid x^{\prime}, z^{\prime}\right)$ and $K^{\Phi}\left(x, z \mid x^{\prime}, z^{\prime}\right)$ in the spectral domain [21], [22], namely, $\tilde{K}_{x x}^{A}\left(k_{\rho} ; z \mid z^{\prime}\right)$ and $\tilde{K}^{\Phi}\left(k_{\rho} ; z \mid z^{\prime}\right)$, being $k_{\rho}=\sqrt{k_{y}^{2}+k_{x}^{2}}=\sqrt{\beta^{2}+k_{x}^{2}}\left(k_{x}\right.$ and $k_{y}$ are the Cartesian spectral variables and $k_{\rho}$ is the radial polar spectral variable). It should be pointed out that if the structure has conductors placed at $N_{l}$ different levels, $\tilde{K}_{x x}^{A}\left(k_{\rho} ; z \mid z^{\prime}\right)$ and $\tilde{K}^{\Phi}\left(k_{\rho} ; z \mid z^{\prime}\right)$ must be evaluated for source and observation points at any of the $N_{l}$ levels. Taking into account the reciprocity of the Green's functions, this lead to $N_{l}\left(N_{l}+1\right) / 2$ combinations of source and observation planes. Once the spectral version of the kernel of our integral equation is known, its 2-D spatial counterpart can be obtained from the following spectral integral:

$$
G\left(x, x^{\prime}\right)=\frac{1}{2 \pi} \int_{-\infty}^{\infty} e^{-j k_{x}\left(x-x^{\prime}\right)} \tilde{G}\left(k_{x}, \beta\right) d k_{x} .
$$

In (4) $G$ and $\tilde{G}$ stand for the spatial (2-D) and spectral representations of any of the $N_{l}\left(N_{l}+1\right) / 2 K_{x x}^{A}$ and $K^{\Phi}$ functions. The $z$-dependence is not explicitly shown since it will not play any role in the development thereafter.

The integrand in (4) may have several poles in the real axis of the $K_{\rho}$-plane, which depend on the structure and frequency.
These poles can be easily removed, as will be explained later on. Another important topological feature of the spectral-domain Green's functions is the existence of branch points at $k_{\rho}= \pm k_{0}$. These branch points are related to the free-space unbounded upper layer of the structure, and they will play an important role in the development of the numerical approach.

The computation of (4) takes a significant part of the overall computation time since the integrands are typically oscillatory and slowly decaying. Since the integrands depend on $\beta$, that integral must be recalculated for every value of the propagation constant in the root search process. Therefore, a fast method to evaluate (4) is of paramount importance. The complex image method already used in the analysis of planar circuits, antennas, and scattering problems [13]-[15], [18] can be adapted to accomplish that goal. The basic idea of this method is to extract from the spectral kernel its quasi-static and surface-wave contributions, and to approximate the remaining function by a sum of complex exponentials. In the 3-D case, the Sommerfeld identity can then be employed to evaluate in closed form the Sommerfeld integrals. This leads to a very efficient algorithm provided that we have a mean to evaluate the quasi-static and surface-wave contributions in closed form. In the 2-D case, as far as the authors know, the spectral integrals in (4) are usually numerically calculated [16], [17]. Although efficient numerical integration algorithms are used, the procedure is not as efficient as those reported in [13]-[15], [18]. What we propose in this paper is to adapt the complex image method to our problem. In order to do this, it is instructive to examine the spectral-domain version of both the vector- and scalar-potential Green's function of a traveling-wave line source in the free space at a height $h$ above a ground plane. These formulas can be written in the following form:

$$
\tilde{G}\left(k_{\rho}\right)=\frac{C_{0}}{u_{0}}\left(1-e^{-2 u_{0} h}\right)
$$

where $u_{0}=\sqrt{k_{\rho}^{2}-k_{0}^{2}},\left(k_{0}=\omega \sqrt{\mu_{0} \varepsilon_{0}}\right), C_{0}=\left(\mu_{0}\right) / 2$ for the $\hat{\boldsymbol{x}} \hat{\boldsymbol{x}}$ component of the dyadic spectral Green's function of the vector potential and $C_{0}=1 /\left(2 \varepsilon_{0}\right)$ for the scalar potential. The first term in (5) corresponds to the effect of the source itself, whereas the second term is the image contribution. Hence, it seems reasonable to think that if the source is embedded in a stratified medium, the spectral Green's function is suitable to be expressed as a quasi-static term $1 /\left(u_{0}\right)$ (which accounts for the near-field contribution of the source and has a singular space-domain counterpart) plus a number of images of the form $A\left(e^{\gamma u_{0}}\right) /\left(u_{0}\right)$. However, it is well known that a stratified medium is also capable of propagating surface waves, which are independent of the source. The influence of these propagating modes in the spectral-domain Green's function is the existence of a finite number of poles that do not appear in the free-space problem. These poles modify the spectral-domain Green's function behavior that can no longer be exclusively expressed as a sum of exponential functions. As a mean to evaluating (4) in a efficient way, we then write the spectral Green's function in the following approximating form:

$$
\tilde{G}\left(k_{\rho}\right)=\tilde{G}_{0}+\tilde{G}_{P}+\frac{1}{u_{0}} \tilde{G}_{\mathrm{GPOF}}
$$


The $\tilde{G}_{0}$ term is the quasi-static contribution, $\tilde{G}_{P}$ represents the surface wave term, and $\tilde{G}_{\mathrm{GPOF}}$ is the remaining term, which is to be expanded by a finite series of complex exponential functions.

\section{A. Quasi-Static Term}

The quasi-static fields are dominant when the distance between the source and field points is small compared to the free-space wavelength. In such a case, the complex exponential appearing in the integrand of (4) oscillates with a large period. Therefore, the behavior of the spatial Green's function is strongly affected by the asymptotic values of the spectral Green's function $\left(k_{x} \gg k_{0}\right)$. Since the integrands decrease very slowly, the following asymptotic behavior for $\tilde{K}_{x x}^{A}\left(k_{\rho} ; z \mid z^{\prime}\right)$ and $\tilde{K}^{\Phi}\left(k_{\rho} ; z \mid z^{\prime}\right)$ must be extracted out:

$$
\begin{aligned}
& \tilde{K}_{x x}^{A}\left(k_{\rho} \rightarrow \infty ; z \mid z^{\prime}\right) \\
& \quad=\frac{\mu_{0}}{2 u_{0}} \times \begin{cases}1, & z=z^{\prime} \\
0, & \text { otherwise }\end{cases} \\
& \tilde{K}^{\Phi}\left(k_{\rho} \rightarrow \infty ; z \mid z^{\prime}\right) \\
& =\frac{1}{2 \varepsilon_{0} u_{0}} \\
& \quad \times \begin{cases}\frac{1}{\sqrt{\varepsilon_{t, n} \varepsilon_{z, n}}}, \quad 2 \\
\frac{\sqrt{\varepsilon_{t, n-1} \varepsilon_{z, n-1}}+\sqrt{\varepsilon_{t, n} \varepsilon_{z, n}}}{\text { otherwise }}, \quad z=z^{\prime} \\
0, \quad z=z_{n}\end{cases}
\end{aligned}
$$

where $z_{n}$ is the $z$ position of the $n$th interface (see Fig. 1). On the other hand, $\varepsilon_{t, i}$ and $\varepsilon_{z, i}$ are the relative permittivities of the $i$ th layer in directions perpendicular and parallel to the $z$-axis, respectively. Note that we will have nonzero asymptotic terms only when source and observation points are at the same level. From (7) and (8), we can, in general, write

$$
\tilde{G}_{0}\left(k_{\rho}\right)=\frac{\kappa C_{0}}{u_{0}}
$$

where $\kappa$ is a constant that is zero if $z \neq z^{\prime}$ and whose value depends on the cases treated in (7) and (8) if source and observation points are at the same level $\left(z=z^{\prime}\right)$. The constant $C_{0}$ was defined in (4).

It should be pointed out that the branch points appearing in $k_{\rho}= \pm k_{0}$ in the spectral Green's functions are also present in $\tilde{G}_{0}$ (9). Therefore, this term does not introduce any new branch cut in the $k_{\rho}$-plane topology [18]. In order to calculate the 2-D space-domain version of (9), the following spectral integral must be carried out:

$$
G_{0}\left(\left|x-x^{\prime}\right|\right)=\frac{\kappa C_{0}}{2 \pi} \int_{-\infty}^{\infty} \frac{e^{-j k_{x}\left|x-x^{\prime}\right|}}{\sqrt{k_{x}^{2}+\alpha^{2}}} d k_{x}
$$

where $\alpha^{2}=\beta^{2}-\omega^{2} \mu_{0} \varepsilon_{0}$. $\alpha$ is supposed to be positive since we are interested only in the bound regime (as opposite to the leaky regime). The integral (10) can be analytically calculated by using the following result [23]:

$$
\begin{aligned}
\int_{-\infty}^{\infty} \frac{e^{-\gamma \sqrt{k_{x}^{2}+\alpha^{2}}}}{\sqrt{k_{x}^{2}+\alpha^{2}}} e^{-j k_{x}\left|x-x^{\prime}\right|} d k_{x} \\
=2 K_{0}\left(\alpha \sqrt{\left(x-x^{\prime}\right)^{2}+\gamma^{2}}\right)
\end{aligned}
$$

where $K_{0}(\cdot)$ is the zeroth-order modified Bessel function of the second kind. The integral in (10) can be seen as the limit of (11) when $\gamma \rightarrow 0$, hence, the contribution of the quasi-static term in the space domain is

$$
G_{0}\left(\left|x-x^{\prime}\right|\right)=\frac{\kappa C_{0}}{\pi} K_{0}\left(\alpha\left|x-x^{\prime}\right|\right) .
$$

Note that since $K_{0}(x) \sim-\ln x$ for small $x$, the 2-D spacedomain Green's functions have a logarithmic singularity when $z=z^{\prime}$ and the field point approaches the source point $\left(x \rightarrow x^{\prime}\right)$.

\section{B. Surface-Wave Poles Contribution}

The complex image scheme can be applied over a complex path, thus avoiding problems related to the presence of poles on the real axis of the complex $k_{\rho}$ plane [24]. However, there are theoretical and numerical reasons that make it advisable to remove the pole contributions from the spectral Green's functions. Complex exponential functions cannot reproduce accurately in the spectral domain the behavior associated to these poles. Since the spectral functions are even functions of $k_{\rho}$, the poles always appear in pairs. Consequently, we can write [14], [15]

$$
\tilde{G}_{P}\left(k_{\rho}\right)=\sum_{p=1}^{N_{0}} \frac{2 R_{p} k_{\rho p}}{k_{\rho}^{2}-k_{\rho p}^{2}}
$$

where $N_{0}$ is the number of poles, $k_{\rho p}$ is the location of the $p$ th pole in the $k_{\rho}$-plane, and $R_{p}$ is its residue

$$
R_{p}=\lim _{k_{\rho} \rightarrow k_{\rho p}} \tilde{G}\left(k_{\rho}\right)\left(k_{\rho}-k_{\rho p}\right) .
$$

Therefore, the space-domain contribution of the surface wave poles is

$$
G_{P}\left(\left|x-x^{\prime}\right|\right)=\frac{1}{2 \pi} \sum_{p=1}^{N_{0}} 2 R_{p} k_{\rho p} \int_{-\infty}^{\infty} \frac{e^{-j k_{x}\left|x-x^{\prime}\right|}}{k_{x}^{2}+\delta_{p}^{2}} d k_{x}
$$

where $\delta_{p}^{2}=\beta^{2}-k_{\rho p}^{2}$ is supposed to be positive (bound regime). An analytical expression for the integral in (14) is available from [23], in such a way that

$$
G_{P}\left(\left|x-x^{\prime}\right|\right)=\sum_{p=1}^{N_{0}} \frac{R_{p} k_{\rho p}}{\delta_{p}} e^{-\delta_{p}\left|x-x^{\prime}\right|} .
$$

Note that, in contrast with the 3-D case [13], [14], the contribution from the surface-wave poles in our 2-D situation does not introduce any singularity. Thus, the treatments reported in [25] or [26] to deal with this problem is not required in our case. Therefore, we can directly extract the surface-wave contribution from the complex image expansion, obtaining a well-behaved approximation for any value of the spatial variable. This makes 
an important difference between the transmission and radiation problems.

\section{Application of the Complex Image Method}

We have found closed-form expressions that allow us to extract the asymptotic and surface wave terms of the spectral-domain Green's function and to recover them in the 2-D space domain. The remaining spectral - domain function is now suitable to be expanded as a finite sum of complex exponential functions by using, for instance, the general pencil of function (GPOF) method [27]

$$
\tilde{G}_{\mathrm{GPOF}}=u_{0}\left(\tilde{G}-\tilde{G}_{0}-\tilde{G}_{P}\right)=\sum_{i=1}^{N_{m}} a_{i} e^{-\gamma_{i} u_{0}}
$$

where $N_{m}$ is the number of employed complex images. It is expected that a short number of images is enough for practical purposes because of the suitability of the expanding functions: they are the spectral version of cylindrical waves.

In order to obtain the unknown coefficients $a_{i}$ and $\gamma_{i}$ appearing in (16), we have sampled our spectral functions on a path in the $k_{\rho}$-plane, which avoids the poles and branch point singularity. To ensure an optimum result, we have applied a two-step procedure that makes it possible to take more samples near the origin, where, owing to the proximity of the branch point, the spectral function presents fast variations. This two-step approach has been proposed by Aksun in [24] for approximating 3-D Green's functions (although this author does not extract the surface-wave contribution). The use of the two-step algorithm is also advantageous to deal with structures having very thin layers. The reasons for that are explained in [28] in the context of the quasi-static analysis of coplanar waveguide (CPW) structures. On the other hand, it is worth noting that although we could also expand the $\tilde{G}_{\text {GPOF }}$ term as a sum of complex exponentials in the $u_{t, i}=\sqrt{k_{\rho}^{2}-k_{0}^{2} \varepsilon_{t, i}}$ or $u_{z, i}=\sqrt{\left(\varepsilon_{t, i}\right) /\left(\varepsilon_{z, i}\right) k_{\rho}^{2}-k_{0}^{2} \varepsilon_{t, i}}$ variables, this would introduce a new branch point in the $k_{\rho}$ plane topology and, therefore, the expansion would fail to approximate the actual function in a correct way [18].

Now, the last term in (6) can be transformed into the 2-D spatial domain by using (11). Therefore, we can write the following expression for the whole 2-D space domain Green's function:

$$
\begin{aligned}
G\left(\left|x-x^{\prime}\right|, \beta\right)= & \frac{\kappa C_{0}}{\pi} K_{0}\left(\alpha\left|x-x^{\prime}\right|\right) \\
& +\sum_{i=1}^{N_{m}} a_{i} K_{0}\left(\alpha \sqrt{\left(x-x^{\prime}\right)^{2}+\gamma_{i}^{2}}\right) \\
& +\sum_{p=1}^{N_{0}} \frac{R_{p} k_{\rho p}}{\delta_{p}} e^{-\delta_{p}\left|x-x^{\prime}\right|} .
\end{aligned}
$$

The 2-D spatial-domain Green's functions are obtained as a sum of radial waves (plus the surface-wave contribution) in the same way as spherical waves are obtained for the spatial-domain 3-D Green's functions [13]. Note that (17) has the important advantage of being an explicit function of the propagation constant $\beta$ through $\alpha$ and $\delta_{p}\left(p=1, \cdots, N_{0}\right)$. In the root searching process involved in the resolution of the eigenvalue problem, the propagation constant $\beta$ is changed many times, but those changes are automatically taken into account by (17). If a numerical integration scheme is applied to solve for (4), the integrals have to be recomputed for each new guess value of $\beta$. The use of our approach obviously implies important central processing unit (CPU) time savings.

\section{APPLICATION OF GALERKIN's METHOD}

Once the space-domain kernel of the integral equation has been efficiently obtained, we can apply the Galerkin's method. A well-established set of basis functions for planar-type structures has been chosen. In fact, for a planar strip of width $w$ and whose central point coordinate is $x_{0}$, the basis functions for the components of the current density have been taken to be

$$
\begin{aligned}
& J_{x}(x)=\sum_{j=0}^{n_{f}-1} I_{j}^{x} \sqrt{1-\left(\frac{x-x_{0}}{w / 2}\right)^{2}} U_{j}\left(\frac{x-x_{0}}{w / 2}\right) \\
& J_{y}(x)=\sum_{j=0}^{n_{f}} I_{j}^{y} \frac{T_{j}\left(\frac{x-x_{0}}{w / 2}\right)}{\sqrt{1-\left(\frac{x-x_{0}}{w / 2}\right)^{2}}}
\end{aligned}
$$

where $n_{f}$ is the number of basis functions employed for the transverse component of the current density $J_{x}$ (one more basis function must be used for the axial component of the current density $J_{y}$ to ensure that the total current fulfills the continuity equation). $T_{j}(\cdot)$ and $U_{j}(\cdot)$ stand for first- and second-kind Chebyshev polynomials, respectively. These functions mimic the real behavior of the currents near the edge of the conducting strips and are quasi- orthogonal for the space kernel we are using. This allows us to attain accurate results while using very few basis functions.

The next step is to calculate the convolution and inner product integrals by using the basis functions in (18) and (19) and the kernel in (17). Thanks to the relationship between first- and second-kind Chebyshev polynomials [29], we only need to compute integrals involving first-kind polynomials. The function $K_{0}$ presents a logarithmic singularity that might cause problems in the convolution integral. Fortunately, the contribution to the convolution integrals of this singularity can be handled in closed form, such as explained in [1]. The rest of the kernel is regular and does not generate integration problems. Due to the type of singularity present in the basis functions, low-order Chebyshev quadratures are suitable to accurately carry out the integrations involving the regular part of the kernel. In this way, the elements of Galerkin's matrix are generated both very accurately and efficiently.

\section{NUMERICAL RESULTS}

The first step for checking the performance of the proposed approach is to verify that the approximation for the 2-D space-domain Green's functions is correct. These functions show an exponential decay with the distance between source and field points, which is quite different from that obtained in the 3-D case. This decaying is faster for large values of $\beta$. 
We have compared in this paper the space-domain Green's functions computed by direct integration a combination of the Romberg's method, and the weighted averages method reported in [11] has been used against those obtained by using the technique in this paper. In this way, Fig. 2 shows $K^{\Phi}$ for the two-layer structure depicted in the figure. The relative difference between numerical and GPOF results is plotted. The GPOF has been applied with and without pole extraction (but always with extraction of the quasi-static term). For the 9-GHz case [see Fig. 2(a)], we can see that the GPOF approximation is very accurate (but fairly better if pole extraction is applied) in the whole range of interest. Relative error is large only for those regions where the values of the approximated function is negligible. In Fig. 2(b), similar data are plotted for a frequency of $33 \mathrm{GHz}$ (two poles are involved in this case). Note that if poles are not removed, large errors are obtained, while a very good approximation is achieved after removing them. Therefore, removing the poles is strongly advised, after all, it is neither difficult nor time consuming to find them [30], while numerical benefits are important. It is worth mentioning that the example considered in Fig. 2 corresponds to a configuration having a very thin dielectric layer. This could cause serious numerical problems, which have been overcome thanks to the application of the two-step scheme used in our study. Although we have concentrated our attention on $K^{\Phi}$, similar conclusions are valid for $K_{x x}^{A}$. This study has been carried out for many combinations of substrates and source and field point locations (coplanar and noncoplanar). The overall conclusion is that the two-step approach in conjunction with the quasi-static term and pole extraction provide an excellent space-domain representation of the required 2-D Green's functions.

Once we are certain about the accuracy of the 2-D space-domain Green's functions computed via (6), we have evaluated the global performance of our method. First of all, we have checked the accuracy and convergence properties of the reaction integrals defining the entries of Galerkin's matrix. We have confirmed that these entries are computed with extreme accuracy (more than six correct figures) using very low-order Chebyshev quadratures and closed-form evaluation of the logarithmic singularity contribution. On the other hand, we have carried out exhaustive comparisons with propagation constants computed using numerical evaluation of the Green's function and using enhanced versions of the SDA [31]. The agreement between the various results is total and we only detect differences in the computational effort (CPU time). It has been verified that pole extraction is necessary for many cases because otherwise the error in the space Green's functions meaningfully affects the final result for the propagation constants. As an example, some numerical results for the fundamental and first two higher order modes of a simple microstrip line are included in Table I. Those results have been obtained by using four basis functions for the longitudinal current and three functions for the transverse one (three and two are enough for the fundamental mode) with and without pole extraction. Extraction of poles is clearly necessary for frequencies above $35 \mathrm{GHz}$. Otherwise results are not reliable because they are strongly dependent on the number of images, sample points, and quadrature points. Moreover, spurious solu-

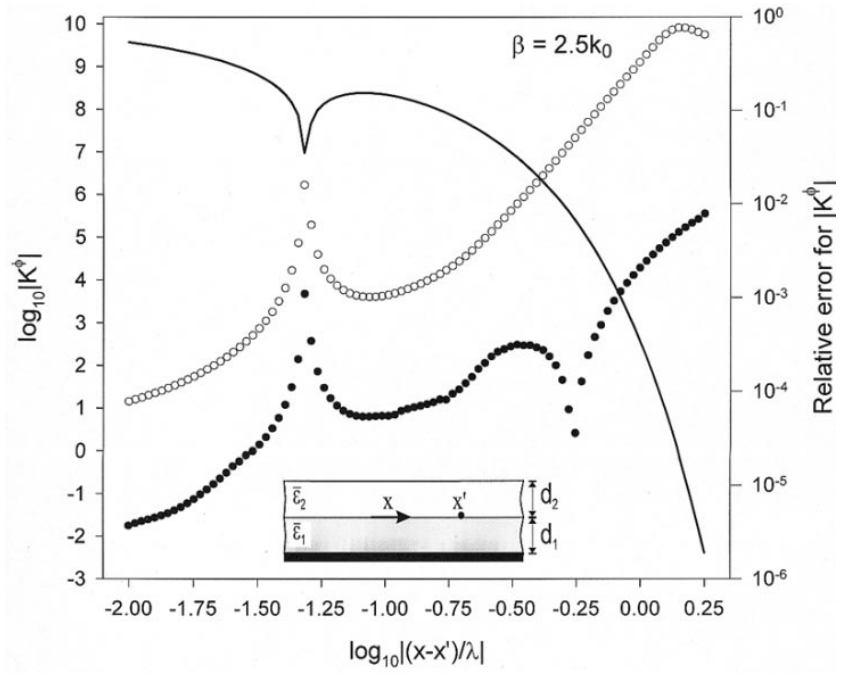

(a)

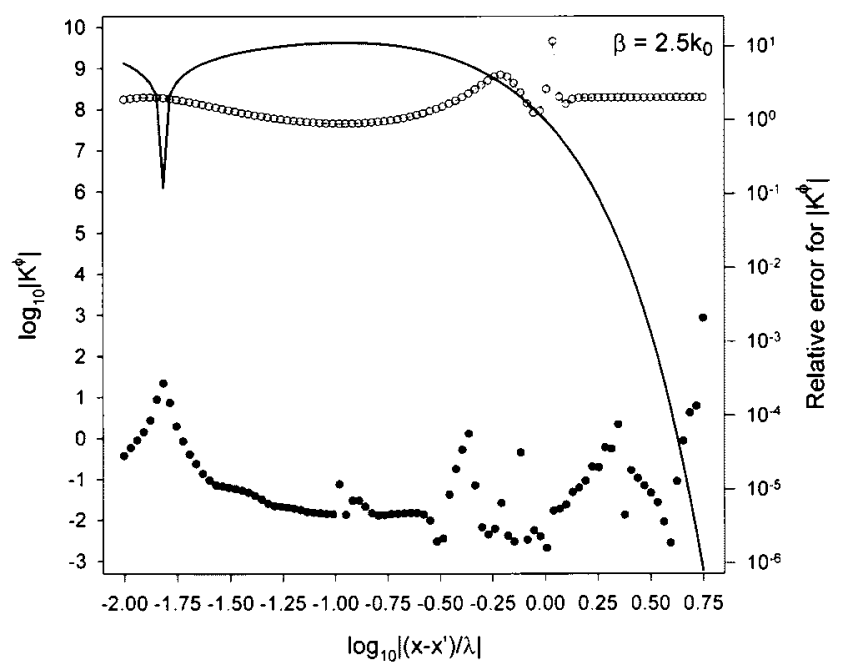

(b)

Fig. 2. Magnitude of $K^{\Phi}$ (solid line) and relative difference between numerical integration computation and complex images computation with (black dots) and without (white dots) pole extraction for: (a) $9 \mathrm{GHz}$ and (b) 33 GHz. Data: $d_{1}=1 \mathrm{~mm}, d_{2}=0.01 \mathrm{~mm}, \varepsilon_{t 1}=\varepsilon_{z 1}=10, \varepsilon_{t 2}=2.25$, and $\varepsilon_{z 2}=1.5$

TABLE I

$\beta / k_{0}$ FOR THE FUNDAMENTAL AND TwO

FIRST HigHER MODES OF THE MiCROSTRIP IN THE TOP FIGURE. $w=3.0 \mathrm{~mm}$ $h=0.635 \mathrm{~mm}$, AND $\varepsilon_{r}=9.8$. LEFT-HAND-SIDE COLUMN: WITH SURFACE

Pole Extraction. Right-HAND-Side COLUMN: No POLE EXTRACTION

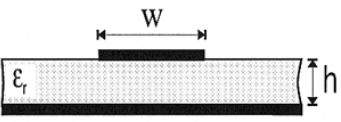

\begin{tabular}{c|c|c||c|c||c|c}
\hline$f(\mathrm{GHz})$ & \multicolumn{2}{|c||}{$E H_{0}$} & \multicolumn{2}{c||}{$E H_{1}$} & \multicolumn{2}{c}{$E H_{2}$} \\
\hline \hline 5 & 2.835 & 2.835 & - & - & - & - \\
10 & 2.895 & 2.895 & - & - & - & - \\
15 & 2.942 & 2.943 & 1.289 & 1.290 & - & - \\
20 & 2.977 & 2.979 & 2.066 & 2.065 & - & - \\
25 & 3.006 & 3.020 & 2.395 & 2.396 & - & - \\
30 & 3.026 & 3.028 & 2.579 & 2.581 & 1.334 & 1.333 \\
35 & 3.042 & 2.981 & 2.696 & 2.701 & 1.818 & 1.817 \\
40 & 3.054 & 2.900 & 2.778 & 2.829 & 2.110 & 2.090 \\
45 & 3.064 & 3.011 & 2.836 & 2.901 & 2.306 & 2.384 \\
50 & 3.071 & 3.032 & 2.881 & 2.996 & 2.445 & 2.558 \\
\hline
\end{tabular}


tions may appear. Eight complex images in the approximation of the Green's functions and four quadrature points in the evaluation of the reaction integrals have been used in this table.

In order to illustrate CPU time saving, we have compared the technique proposed in this paper with numerical evaluation of the space-domain Green's function. Even though the integration scheme we have used to generate the numerical samples of the Green's function is very efficient, the application of the method proposed here still yields an important reduction of CPU time. Moreover, complex images have not to be recomputed when $\beta$ is changed in the root searching process, whereas new numerical integrations would be required for new values of $\beta$. Hence, the relative impact of using this approach in the analysis of transmission lines (eigenvalue 2-D problem) is probably stronger than the impact of using a similar technique in a 3-D planar problem since, in the latter case, the generation of the Green's functions is a small fraction of the total numerical effort. As an example, Fig. 3 shows the ratio of CPU times using numerical integration against the method reported here as a function of the number of strips (the same number of basis functions has been used in each strip so as to keep the same accuracy level). CPU time reduction is significant for any case, becoming more important as the complexity of the multistrip system increases.

As a final example, Fig. 4 shows the dispersion curves for the fundamental modes of the five conductor microstrip transmission line depicted in the figure. Two cases are considered: in Case A, the strips are in the air-dielectric interface; in Case $\mathrm{B}$, the center conductor resides on the top interface of a very thin cover layer. Dispersion curves of the configuration (A) were published by Kitazawa in [32] and later reproduced by Hsu in [33]. Results for the configuration B are given in [33]. Kitazawa uses a variational method, whereas Hsu employs an MPIE scheme solved in the space domain by using the method of moments with piecewise linear basis functions and numerical computation of the spectral integrals. The agreement between our results and those presented in [32] and [33] is very good, as can been seen in the graphical representation. In order to reproduce those data, we have used three longitudinal and two transverse basis functions along with four quadrature points and eight complex images for approximating the regular part of the spectral-domain Green's functions. Many other results reported in the literature have been reproduced with our method, but they are not included here for the sake of brevity.

As a final comment on the accuracy and robustness of the proposed method, we have to say that very accurate results are also obtained for the current distribution. A systematic increase of the number of basis functions does not introduce numerical instabilities and all the coefficients of the current expansion are computed with very good accuracy (five correct figures are easily obtained for the expansion coefficients). This is more significant regarding the quality of the employed technique than propagation constant results [9]. To sum up, our many numerical experiments confirm that the developed method works properly, providing very accurate results and important computational-effort savings.

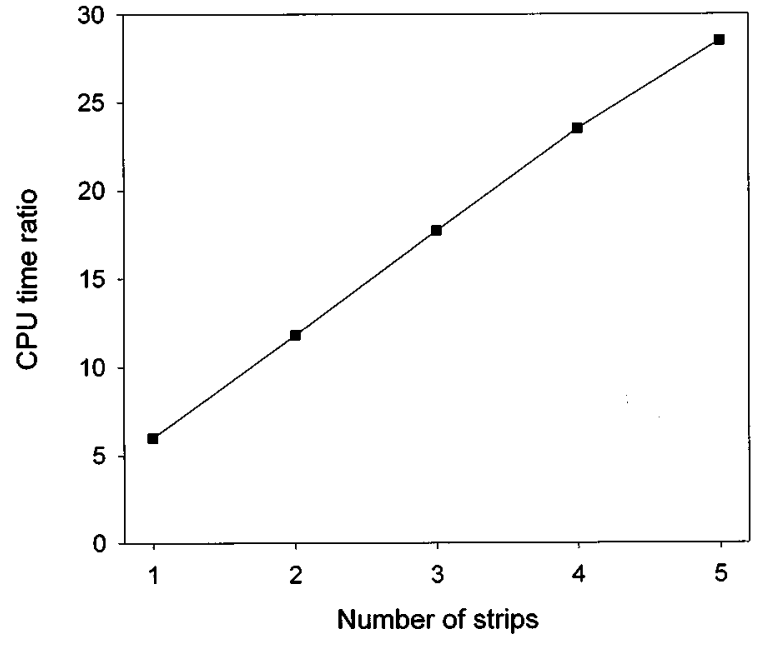

Fig. 3. CPU time ratio for a microstrip analysis using numerical generation of the Green's function and the technique in this paper as a function of the number of strips. Longitudinal and transverse currents have been approximated by means of three and two basis functions, respectively. Substrate: thickness $=0.635 \mathrm{~mm}, \epsilon_{r}=9.8$. Strip width $=3 \mathrm{~mm}$. Strip separation: $1.5 \mathrm{~mm}$.

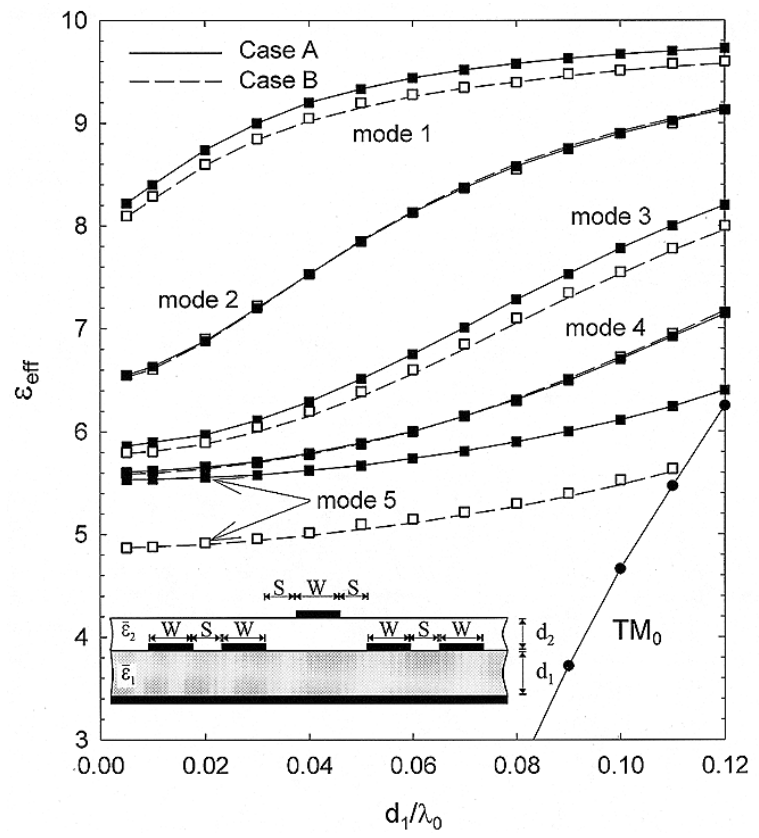

Fig. 4. Dispersion curves of the five fundamental modes of the structure of the figure. Dielectric data as in Fig. $2 . w=1 \mathrm{~mm}, s=0.2 \mathrm{~mm}$. Case (A): $d_{2}=0$. Black squares: results in [32] and [33], solid line: our results. Case (B): $d_{2}=0.01 d_{1}$. White squares: results in [33], dash line: our results.

\section{CONCLUSIONS}

A new method has been proposed for the computation of the dispersion curves of multilevel multiconductor planar transmission lines embedded in a uniaxially anisotropic stratified medium. The approach is based on the complex image technique and MPIE formulation. We take advantage of a closed-form derivation of the 2-D space-domain Green's function and of the use of a suitable set of basis functions to obtain a fast and accurate computer code. The evaluation of Galerkin's matrix entries is performed in a very efficient way. Numerical results have 
been presented and compared with data available in the literature and supplied by other methods. Very good agreement has been found in all cases by using very modest computational resources.

\section{REFERENCES}

[1] J. Bernal, F. Medina, and M. Horno, "Quick quasi-TEM analysis of multiconductor transmission lines with rectangular cross section," IEEE Trans. Microwave Theory Tech., vol. 45, pp. 1619-1626, Sept. 1997.

[2] Y. Huang and S. -L. Lai, "Regular solution of shielded planar transmission lines," IEEE Trans. Microwave Theory Tech., vol. 42, pp. 84-91, Jan. 1994.

[3] Y. -S. Xu and A. S. Omar, "Rigorous solution of mode spectra for shielded multilayer microstrip lines," IEEE Trans. Microwave Theory Tech., vol. 42, pp. 1213-1222, July 1994.

[4] T. Rozzi, L. Pierantoni, and M. Farina, "Eigenvalue approach to the efficient determination of the hybrid and complex spectrum of inhomogeneous, closed waveguide," IEEE Trans. Microwave Theory Tech., vol. 45, pp. 345-353, Mar. 1997.

[5] G. A. Kyriacou and J. N. Sahalos, "A Wiener-Hopf-type analysis of uniaxial substrate-superstrate microstrip structures," IEEE Trans. Microwave Theory Tech., vol. 45, pp. 616-629, May 1997.

[6] K. Uchida, T. Noda, and T. Matsunaga, "New type of spectral domain analysis of a microstrip line," IEEE Trans. Microwave Theory Tech., vol. 37, pp. 947-952, June 1989.

[7] G. Cohen, N. Fach, and D. De Zutter, "Comparison between two sets of basis functions for the current modeling in the Galerkin spectral domain solution for microstrips," IEEE Trans. Microwave Theory Tech., vol. 42, pp. 505-513, Mar. 1994.

[8] S. Park and C. A. Balanis, "Dispersion characteristics of open microstrip lines using closed form asymptotic extraction," IEEE Trans. Microwave Theory Tech., vol. 45, pp. 458-460, Mar. 1997.

[9] G. Cano, F. Medina, and M. Horno, "On the efficient implementation of SDA for boxed strip like and slot like structures," IEEE Trans. Microwave Theory Tech., vol. 46, pp. 1801-1806, Nov. 1998.

[10] A. W. Glisson and D. R. Wilton, "Simple and efficient numerical methods for problems of electromagnetic radiation and scattering from surfaces," IEEE Trans. Antennas Propagat., vol. AP-28, pp. 593-603, Sept. 1980

[11] J. R. Mosig and F. E. Gardiol, "Analytic and numerical techniques in the Green's function treatment of microstrip antennas and scatterers," Proc. Inst. Elec. Eng., vol. 130, pp. 175-182, Mar. 1983.

[12] K. A. Michalsky, "The mixed potential electric field integral equation for objects in a layered media," Arch. Elektr. Üerbertrag. Tech., vol. 39, pp. 317-322, Sept./Oct. 1985

[13] D. G. Fang, J. J. Yang, and G. Y. Delisle, "Discrete image theory for horizontal electric dipoles in a multilayered medium," Proc. Inst. Elect. Eng., pt. H, vol. 135, pp. 297-303, Oct. 1988.

[14] Y. L. Chow, J. J. Yang, D. G. Fang, and G. E. Howard, "A closed form spatial Green's function for the thick microstrip substrate," IEEE Trans. Microwave Theory Tech., vol. 39, pp. 588-592, Mar. 1991

[15] M. I. Aksun and R. Mittra, "Derivation of closed form Green's functions for a general microstrip geometry," IEEE Trans. Microwave Theory Tech., vol. 40, pp. 2055-2061, Nov. 1992.

[16] K. A. Michalski and D. Zheng, "Rigorous analysis of open microstrip lines of arbitrary cross section in bound and leaky regimes," IEEE Trans. Microwave Theory Tech., vol. 37, pp. 2005-2010, Dec. 1989.

[17] C-I. G. Hsu, R. F. Harrington, K. A. Michalski, and D. Zheng, "Analysis of multiconductor transmission lines of arbitrary cross section in multilayered uniaxial media," IEEE Trans. Microwave Theory Tech., vol. 41, pp. 70-78, Jan. 1993.

[18] R. A. Kipp and C. H. Chan, "Complex image method for sources in bounded regions of multilayer structures," IEEE Trans. Microwave Theory Tech., vol. 42, pp. 860-865, May 1994.

[19] J. R. Mosig, "Arbitrarily shaped microstrip structures and their analysis with a mixed potential integral equation," IEEE Trans. Microwave Theory Tech., vol. 36, pp. 314-323, Feb. 1988.

[20] A. Sommerfeld, Partial Differential Equations in Physics, New York: Academic, 1949.

[21] K. A. Michalski and D. Zheng, "Electromagnetic scattering and radiation by surfaces of arbitrary shape in layered media-Part I: Theory," IEEE Trans. Antennas Propagat., vol. 38, pp. 335-344, Mar. 1990.

[22] L. B. Felsen and N. Marcuvitz, Radiation and Scattering of Waves. Englewood Cliffs, NJ: Prentice-Hall, 1973.
[23] I. S. Gradshteyn and I. M. Ryzhik, Table of Integrals, Series, and Products. New York: Academic, 1980

[24] M. I. Aksun, "A robust approach for the derivation of closed form Green's functions," IEEE Trans. Microwave Theory Tech., vol. 44, pp. 651-658, May 1996.

[25] C. H. Chan and R. A. Kipp, "Application of the complex image method to multilevel, multiconductor microstrip lines," Int. J. Microwave Millimeter wave CAE, vol. 7, pp. 359-367, Sept. 1997.

[26] F. J. Demuynck, A. E. Vandenbosch, and A. R. Van de Capelle, "The expansion wave concept-Part I: Efficient calculation of spatial Green's functions in a stratified dielectric medium," IEEE Trans. Antennas Propagat., vol. 46, pp. 397-406, Mar. 1998.

[27] T. K. Sarkar and O. Pereira, "Using the matrix pencil method to estimate the parameters of a sum of complex exponentials," Antennas Propagat. Mag., vol. 37, pp. 48-55, Feb. 1995.

[28] J. Bernal, F. Medina, and M. Horno, "Quasi-static analysis of multiconductor CPW by using the complex images method," Int. J. RF Microwave Computer-Aided Eng., vol. 8, no. 5, pp. 405-416, 1998.

[29] G. Arfken, Mathematical Methods for Physicists. New York: Academic, 1985.

[30] F. Mesa and M. Horno, "Computation of proper and improper modes in multilayered bianisotropic waveguides," IEEE Trans. Microwave Theory Tech., vol. 43, pp. 233-235, Jan. 1995.

[31] F. Mesa, R. Marques, and M. Horno, "An efficient numerical spectral domain method to analyze a large class of nonreciprocal planar transmission lines," IEEE Trans. Microwave Theory Tech., vol. 40, pp. 1630-1641, Aug. 1992.

[32] T. Kitazawa, "Variational method for multiconductor coupled striplines with stratified anisotropic media," IEEE Trans. Microwave Theory Tech., vol. 37, pp. 484-491, Mar. 1989.

[33] C. G. Hsu, "Analysis of a multiconductor transmission line embedded in a layered uniaxial medium using a mixed potential integral equation approach," Ph.D. dissertation, Dept. Elect. Eng., Syracuse Univ., Syracuse, NY, Sept. 1991.

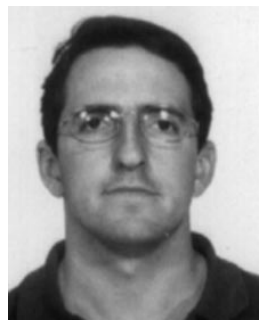

Joaquin Bernal was born in Seville, Spain, in 1971. He received the Licenciado degree in physics from the University of Seville, Seville, Spain, in 1994, and is currently working toward the Ph.D. degree at the University of Seville.

In 1995, he joined the Department of Electronic and Electromagnetism, University of Seville. Since 1998, he has been an Assistant Professor in the Department of Applied Physics, University of Seville. His research interests focus on the analysis of planar structures for integrated microwave circuits.

Mr. Bernal was the recipient of a 1995 scholarship presented by Junta de Andalucía.

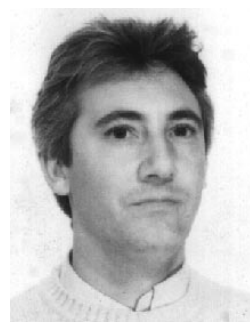

Francisco Medina (M'90) was born in Puerto Real, Cádiz, Spain, in November 1960. He received the Licenciado and the doctor degrees from the University of Seville, Seville, Spain, in 1983 and 1987, respectively, both in physics.

From 1986 to 1987, he spent the academic year at the Laboratoire de Microondes de l'ENSEEIHT, Toulouse, France.

From 1985 to 1989, he was a Profesor Ayudante with the Department of Electronics and Electromagnetism, University of Seville, and since 1990, he has been Profesor Titular of electromagnetism. He is currently the Head of the Microwaves Group, University of Seville. His research interests include analytical and numerical methods for planar structures and circuits and the influence on these circuits of anisotropic materials.

Dr. Medina was a member of the Technical Programme Committee of the 23rd European Microwave Conference, Madrid, Spain (1993). He is on the editorial board of the IEEE TRANSACTIONS ON MICROWAVE THEORY AND TECHNIQUES. He was the recipient of a Ministerio de Educacion y Ciencia/Ministere de la Recherche et la Technologie Scholarship. 


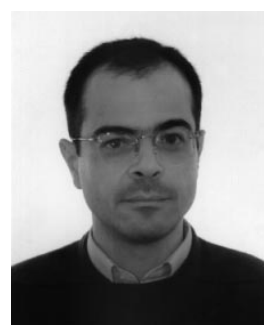

Rafael R. Boix (M'97) was born in Melilla, Spain, in 1962. He received the Licenciado and doctor degrees in physics from the University of Seville, Seville, Spain, in 1985 and 1990, respectively.

Since 1985, he has been with the Electronics and Electromagnetics Department, University of Seville, where he became an Associate Professor in 1994. During the summers of 1991 and 1992, he was with the Electrical Engineering Department, University of California at Los Angeles, as a Visiting Scholar. During the summer of 1996, he was with the Electrical and Computer Engineering Department, Syracuse University, Syracuse, NY, as a Visiting Scholar. His current research interest is focused on the analysis of the effects of complex substrates on the performance of planar transmission-line discontinuities, planar passive microwave circuits, planar resonators, and printed circuit antennas.

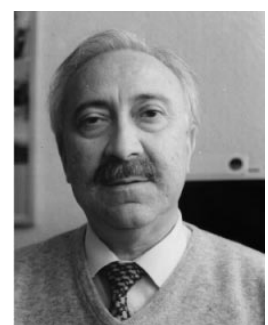

Manuel Horno (M'75) was born in Torre del Campo, Jaén, Spain, and died in September 1998, in Seville, Spain. He received the Licenciado and the doctor degrees from the University of Seville, Spain, in 1969 and 1972, respectively, both in physics.

In October 1969, he joined the Department of Electronics and Electromagnetism, University of Seville, where he became an Assistant Professor in 1970, Associate Professor in 1975, and Professor in 1986. His main fields of interest included boundary value problems in electromagnetic theory, wave propagation through anisotropic media, and microwave integrated circuits. During his final years, he was engaged in the analysis of planar transmission lines embedded in anisotropic materials, multiconductor transmission lines, and planar antennas. He was a member of the Electromagnetism Academy, Massachusetts Institute of Technology (MIT), Cambridge. 